\title{
Antinociceptive activity of a synthetic oxopyrrolidine-based compound, ASH21374, and determination of its possible mechanisms
}

\begin{abstract}
This study was carried out to determine the antinociceptive activity of a novel synthetic oxopyrrolidine-based compound, (2R,3R,4S)-ethyl 4-hydroxy-1,2-dimethyl-5oxopyrrolidine-3-carboxylate (ASH21374), and to elucidate the involvement of the opioid, vanilloid, glutamate, and nitric oxide - cyclic guanosine monophosphate (NO/cGMP) systems in modulating the observed antinociception. ASH21374, in the doses of 2, 10, and $100 \mathrm{mg} / \mathrm{kg}$ body mass, was administered orally to mice 60 mins prior to exposure to various antinociceptive assays. From the results obtained, ASH21374 exhibited significant $(\mathrm{P}<0.05)$ antinociceptive activity in the abdominal constriction, hot-plate, and formalin tests that was comparable with $100 \mathrm{mg} / \mathrm{kg}$ acetylsalicylic acid or $5 \mathrm{mg} / \mathrm{kg}$ morphine, respectively. ASH21374 also attenuated capsaicin- and glutamate-induced paw licking. Pre-treatment with $5 \mathrm{mg} / \mathrm{kg}$ naloxone significantly $(\mathrm{P}<0.05$ ) inhibited the activity in all assays, while pretreatment with $10 \mathrm{mg} / \mathrm{k}$-funaltraxamine, $1 \mathrm{mg} / \mathrm{kg}$ naltrindole, or $1 \mathrm{mg} / \mathrm{kg}$ norbinaltorphimine significantly $(\mathrm{P}<0.05)$ reversed the activity in the abdominal constriction test. L-Arginine,NG-nitro-L-arginine methyl esters (L-NAME), methylene blue, and their combinations, failed to inhibit the ASH21374 antinociceptive activity. In conclusion, ASH21374 demonstrated antinociceptive activities on the peripheral and central nervous systems, mediated through the activation of opioid receptors, inhibition of the glutamatergic system, and attenuation of vanilloid-mediated nociceptive transmission. Further studies have been planned to determine the pharmacological potential of ASH21374.
\end{abstract}

Keyword: ASH21374; Oxopyrrolidine; Antinociceptive; Opioid; Glutamatergic 